\title{
A THEOREM OF DICKSON ON NONVANISHING CUBIC FORMS IN A FINITE FIELD
}

\section{CARLITZ}

Dickson stated that a ternary cubic form with coefficients in $G F(q), q=p^{n}, p>2$, that vanishes only for $x=y=z=0$, is the norm of a linear form with coefficients in $G F\left(q^{3}\right)$. The proof [2, p. 161, particularly line 15] does not seem adequate. In this note we sketch another proof.

Let $f=f(x, y, z)$ be a homogeneous cubic polynomial with coefficients in $G F(q)$ that vanishes in $G F(q)$ only for $x=y=z=0$. Put

$$
f=f_{0} z^{3}+f_{1} z^{2}+f_{2} z+f_{3}
$$

where $f_{j}=f_{j}(x, y)$ is homogeneous of degree $j$. Assume first that $f_{0} \neq 0$, so that we may take $f_{0}=1$; also we assume to begin with that $p \neq 3$. Then by means of a linear transformation we may suppose that $f_{1}=0$. Hence consider

$$
f=z^{3}+f_{2} z+f_{3} \text {. }
$$

Now for fixed $a, b \in G F(q), a, b$ not both 0 , it is clear that $f(a, b, z)$ is irreducible. Consequently its discriminant is a nonzero square of $G F(q)$; that is

$$
D=D(a, b)=-4 f_{2}^{3}(a, b)-27 f_{3}^{2}(a, b)
$$

is a square for all $a, b \in G F(q), D=0$ only for $a=b=0$. But Dickson [1, Theorem 3] has proved that for $q \geqq 13$, this implies

$$
D(x, y)=C^{2}(x, y),
$$

where $C(x, y)$ is a homogeneous cubic polynomial. Thus (3) becomes

$$
C^{2}(x, y)=-4 f_{2}^{3}(x, y)-27 f_{3}^{2}(x, y) .
$$

Clearly it is necessary that $f_{3}(x, y)$ be irreducible, for otherwise there exist two number $a, b$ of the field, not both 0 , such that $f_{3}(a, b)$, and therefore $f(a, b, 0)=0$. Thus either $f_{2}=0$ or $\left(f_{2}, f_{3}\right)=\left(C, f_{3}\right)=1$. Leaving the case $f_{2}=0$ for the moment, we rewrite (4) as

$$
C^{2}+27 f_{3}^{2}=-4 f_{2}^{3}
$$

and put $\alpha^{2}=-27$, where $\alpha \in G F\left(q^{2}\right)$. Then clearly

Received by the editors June 5, 1956 and, in revised form, February 18, 1957. 


$$
C+\alpha f_{3}=2 \beta u^{3}, \quad C-\alpha f_{3}=-2 \beta^{-1} v^{3},
$$

where $\beta \in G F\left(q^{2}\right)$ and $u, v$ are linear forms with coefficients in $G F\left(q^{2}\right)$. Since (5) implies $C=\beta u^{3}-\beta^{-1} v^{3}, \alpha f_{3}=\beta u^{3}+\beta^{-1} v^{3}, f_{2}=u v$, (2) becomes

$$
f=z^{3}+u v z+\alpha^{-1} \beta u^{3}+\alpha^{-1} \beta^{-1} v^{3}
$$

or

$$
f=z^{3}-3 U V z+U^{3}+V^{3},
$$

where $U, V$ are linear forms in some $G F\left(q^{k}\right)$. But (6) evidently implies $f(-U-V)=0$, from which it follows that the linear form $U+V$ has coefficients in $G F\left(q^{3}\right)$. Indeed if $-U-V=\lambda x+\mu y$, then

$$
f(x, y, \lambda x+\mu y)=0
$$

implies $f(x, 0, \lambda x)=0$, so that $\lambda \in G F\left(q^{3}\right)$; similarly for $\mu$. Therefore $f$ is the norm of a linear form in $G F\left(q^{3}\right)$.

In the excluded case $f_{2}=0$, we have

$$
f=z^{3}+f_{3}(x, y) .
$$

Since $f_{3}$ is irreducible, it is only necessary to consider the case

$$
f=z^{3}+a x^{3}+b y^{3} \quad(a b \neq 0),
$$

for the occurrence of a term $x^{2} y$ or $x y^{2}$ can be treated as in the previous proof. Next we need only consider the case $q \equiv 1(\bmod 3), a b=1$, $a$ not a cube of $G F(q)$. But by a theorem of Hurwitz [3] which may be extended without difficulty to finite fields, the equation

$$
a x^{3}+b y^{3}+z^{3}=0,
$$

where $a, b$ satisfy the stated conditions, has at least one solution with $x y z \neq 0$.

Returning to (1), suppose that $f_{0}=0$. If a term in $x^{3}$ or $y^{3}$ occurs, the previous proof applies. We need then consider only the case $f_{3}=a x^{2} y+b x y^{2}$, and this obviously leads to a contradiction.

When $p=3$ the above discussion must be modified somewhat. Returning to (1), we may assume $f_{0}=1$ but cannot require that $f_{1}=0$. If indeed $f_{1}=0$, then (4) reduces to

$$
C^{2}(x, y)=-4 f_{2}^{3}(x, y),
$$

so that $C$ is the cube of a linear form; this contradicts the requirement that $C$ vanishes only for $x=y=0$.

Employing the discriminant of (1) we get

$$
-f_{1}^{3} f_{3}-f_{2}^{3}+f_{1}^{2} f_{2}^{2}=C^{2} \text {. }
$$


If $f_{2}=0,(8)$ becomes $-f_{1}^{3} f_{3}=C^{2}$, which contradicts the irreducibility of $f_{3}$. We need then consider only the case $f_{1} f_{2} \neq 0$. By means of a linear transformation we may suppose that $f_{1}=x, f_{2}=a y^{2}$; then (8) reduces to

$$
-a^{3} y^{6}+a^{2} x^{2} y^{4}-x^{3} f_{3}=C^{2} .
$$

We may take $a=-1, C=y^{3}-x^{2} y+c x^{3}$, which yields

$$
f_{3}=c y^{3}-x y^{2}+c x^{2} y+c^{2} x^{3} \text {. }
$$

In (1) take $f_{1}=x, f_{2}=-y^{2}, z=\alpha x+\beta y$. Substituting in $f(x, y, z)=0$, we obtain the following conditions on $\alpha, \beta$ :

$$
\alpha^{3}+\alpha^{2}=c^{2}, \quad \beta^{3}-\beta=c, \quad \alpha \beta=c, \quad \beta^{2}-\alpha=1 .
$$

It is easily verified that this system is equivalent to

$$
\beta^{3}-\beta=c, \quad \alpha \beta=c .
$$

Clearly (9) implies $\alpha, \beta \in G F\left(q^{3}\right)$. Hence $f(x, y, z)$ has the linear factor $z-\alpha x-\beta y$, and it follows at once that

$$
f(x, y, z)=(z-\alpha x-\beta y)\left(z-\alpha^{q} x-\beta^{q} y\right)\left(z-\alpha^{q^{2}} x-\beta^{q^{2}} y\right) .
$$

In other words, $f(x, y, z)$ is the norm of a linear form with coefficients in $G F\left(q^{3}\right)$; moreover $1, \alpha, \beta$ are linearly independent with respect to $G F(q)$.

We may state the following

Theorem. Let $f(x, y, z)$ be a homogeneous cubic polynomial with coefficients in $G F(q)$ that vanishes in $G F(q)$ only for $x=y=z=0$. Then if $p>2, q \geqq 13, f(x, y, z)$ is the norm of a linear form $\alpha x+\beta y+\gamma z$, where $\alpha, \beta, \gamma$ are in $G F\left(q^{3}\right)$ and are linearly independent with respect to $G F(q)$.

The converse of the theorem is evidently true. Possibly the theorem holds for $p=2$; the above proof obviously does not apply in that case.

\section{REFERENCES}

1. L. E. Dickson, Definite forms in a finite field, Trans. Amer. Math. Soc. vol. 10 (1909) pp. 109-122.

2. - On triple algebras and ternary cubic forms, Bull. Amer. Math. Soc. vol. 14 (1907-1908) pp. 160-169.

3. A. Hurwitz, Über die Kongruenz $a x^{e}+b y^{e}+c z^{e} \equiv 0(\bmod p)$, J. Reine Angew. Math. vol. 136 (1909) pp. 272-292 (= Mathematische Werke, vol. 2, Basel, 1933, pp. 430-445).

DUKE UNIVERSITY 